\title{
Efficacy of long-lasting insecticidal nets in use in Macha, Zambia, against the local Anopheles arabiensis population
}

\author{
Laura C Norris ${ }^{*}$ and Douglas E Norris
}

\begin{abstract}
Background: The mosquito Anopheles arabiensis is the primary vector of Plasmodium falciparum in Macha, Zambia. A major portion of Zambia's current malaria control programme relies on long-lasting insecticide-treated nets (LLINS) and indoor residual spraying (IRS) with insecticides. Currently, the efficacy of these measures against An. arabiensis in Macha is unknown, and previous data has shown that An. arabiensis has continued to feed on human hosts, despite high ITN coverage. It is possible that this could be due to either decreased efficacy of ITNs in used in Macha, or pyrethroid resistance in the vector.

Methods: F1 offspring of field-collected adult An. arabiensis were tested for insecticide resistance, using CDC bottle bioassays and deltamethrin ITN susceptibility assays. The mosquitoes were characterized for the knock-down resistance $(k d r)$ allele by PCR. LLINs that had been in use for two years in nearby villages were collected and tested for residual deltamethrin concentration and net quality, and were used in bioassays against susceptible colonized Anopheles gambiae s.s. Keele. Additionally, a survey on ITN use and care was conducted among LLIN owners.

Results: In the F1 An. arabiensis field population, low levels of resistance to DDT and deltamethrin-treated net material were detected by bioassay, although the knock-down resistance $(k d r)$ allele not present in the population. ITN evaluations revealed high variability in residual deltamethrin concentration, quality of the nets, and mosquito mortality in bioassays. Mortality against An. gambiae s.s. in bioassays was correlated with residual deltamethrin concentration, which was dependent upon the number of washes each net had received.

Conclusions: Proper LLIN care was a strong determinant of LLIN efficacy, indicating that education on the importance of LLIN use and care is key when distributing nets. As there is little insecticide resistance in the local vector population, degradation of LLINs most likely allowed for continued human feeding by An. arabiensis. Continued monitoring and assessment of both the vector population and the efficacy of LLINs in use is necessary in order to appropriately modify vector control operations and prevent the development of pyrethroid resistance.
\end{abstract}

\section{Background}

Malaria is a severe public health problem, causing an estimated 225 million disease cases and 781,000 deaths per year [1]. Most victims are children under five years old living in sub-Saharan Africa [1]. Malaria is transmitted by Anopheles mosquitoes, and because there is currently no vaccine available, vector control is one of the most important means of malaria prevention. Insecticide-treated nets (ITNs), introduced over 20 years ago

\footnotetext{
* Correspondence: Inorris@jhsph.edu

W. Harry Feinstone Department of Molecular Microbiology and Immunology, Johns Hopkins Bloomberg School of Public Health, 615 N. Wolfe St.
} Baltimore, MD 21218, USA
$[2,3]$, are an important tool to protect individuals against the morbidity and mortality caused by malaria $[4,5]$. ITNs can also decrease local malaria transmission by mass killing and decreased survival of anopheline vectors, thereby protecting those in the community without ITNs [6]. A more recent innovation is the long-lasting insecticide-treated net (LLIN), in which insecticide is either incorporated into the fiber during extrusion, or coated on the fiber or the finished net with a binding agent. Unlike conventional ITNs, which lose effective insecticide after one or two washes and last only 6-12 months, LLINs retain effective doses of insecticide up to 20 washes and have an expected lifespan of 3 to 4 years 
$[7,8]$. One LLIN recommended by the World Health Organization Pesticide Evaluation Scheme (WHOPES) is Permanet $^{\circledR}$, which contains $55 \mathrm{mg} / \mathrm{m}^{2}$ deltamethrin \pm $25 \%$ (minimum $45 \mathrm{mg} / \mathrm{m}^{2}$ ), and should retain $25 \mathrm{mg} / \mathrm{m}^{2}$ deltamethrin after 6 standard washes, enough to still be effective against mosquitoes [9]. In laboratory tests, it provides $80 \%$ functional mortality for up to 20 laboratory washes [9].

Roll Back Malaria has set targets for global malaria control, including protecting $80 \%$ of at risk populations with ITNs or indoor residual spraying (IRS) by 2010 [10]. Zambia has had great success in rapidly scaling up malaria control measures [11,12]. In 2010, 73\% of all households had at least one ITN or IRS within the last 12 months. Additionally, 52\% of children under five years of age and $46 \%$ of pregnant women reported sleeping under an ITN the previous night [12]

Differences in use and wear in field settings may cause LLINs to lose residual insecticide more quickly, leading to loss of efficacy. A number of studies have examined the dynamics of insecticide loss and LLIN efficacy in field situations, with varying results. In Colombia, Permanet ${ }^{\circledR}$ 1.0 nets that had been washed 23 times over three years retained a mean of $9.6 \mathrm{mg} / \mathrm{m}^{2}$ deltamethrin, and caused 79\% mortality in Anopheles mosquitoes after three minutes of exposure in a WHO cone assay [13]. Similarly, a study in Kenya examined mean time to ITN failure, using $<50 \%$ mortality in WHO cone assays with Anopheles gambiae as a cutoff [14]. After two years of use, $82 \%$ of the Permanet ${ }^{\circledR} 1.0$ LLINs were still effective, and the mean concentration of insecticide in failed nets was $11 \mathrm{mg} / \mathrm{m}^{2}$. In a multi-country trial of Permanet ${ }^{\circledR} 2.0$ LLINs, the nets retained $13-24 \mathrm{mg} / \mathrm{m}^{2}$ deltamethrin after 20 washes, and caused $80 \%$ mortality in Anopheles stephensi [15]. These studies all used standardized washing procedures. In contrast, a study in Ethiopia assayed Permanet ${ }^{\circledR} 2.0$ LLINs used by villagers for two years without intervention. These nets maintained $67-72 \%$ mortality against Anopheles arabiensis [16]. A study of Permanet ${ }^{\circledR}$ 2.0 LLINs in Uganda found 74\% functional mortality after two years of household use in rural conditions [17].

In addition to insecticide loss, LLINs may lose efficacy if insecticide resistance develops in the mosquito target. Overuse of insecticides, whether in ITNs, indoor residual spraying, or in agricultural use, can select for resistance in mosquito vector species [18]. Resistance can be mediated either by mutations in the target site of the insecticide or its active metabolites (target-site resistance), or through enzymatic modification of insecticides to produce non-toxic metabolites (metabolic detoxification). DDT and pyrethroids, two types of insecticide approved for malaria control [19], share a common target, the para voltage-gated sodium channel. Knockdown resistance $(k d r)$ mutations in the gene that codes for this channel can therefore confer cross-resistance to both DDT and pyrethroids that, when combined with metabolic resistance, can compromise insecticide-based malaria control efforts [20]. The $k d r$ mutation has been shown to cause pyrethroid resistance in An. gambiae s.s. [21], and is present in several $A n$. arabiensis populations in Africa: Kenya [22], South Africa [23], Burkina Faso [24], and Ethiopia [25].

Additionally, resistance to DDT and pyrethroids can be mediated through metabolic detoxification, by upregulation of cytochrome P450-dependent monooxygenases and glutathione S-transferases [18]. Phenotypic pyrethroid resistance in $A n$. arabiensis has emerged in the Sudan [26], Mozambique [27], Uganda [28], and in Gwave, Zimbabwe [29], directly across the border from Southern Province, Zambia. DDT resistance in An. arabiensis has emerged in Sudan [26] and South Africa [23].

The Southern Province of Zambia has historically had hyperendemic Plasmodium falciparum malaria transmission [30] vectored primarily by $A n$. arabiensis, with $A n$. funestus as a secondary vector [31]. In the Choma District, where Macha is located, $61-80 \%$ of households have target coverage of 3 ITNs per household [11]. As part of the malaria control scale-up, a free mass distribution of ITNs by the Zambian government provided 4,800 LLINs to the Macha area in 2007, and $75 \%$ of individuals are protected by an ITN [32]. However, the pyrethroid susceptibility status of the $A n$. arabiensis population in Southern Zambia is unknown.

There is $100 \%$ ITN coverage in households in Macha where mosquito surveillance collections are performed, yet 25-28\% of An. arabiensis collected in CDC light traps are engorged, with human blood indices of 94-96\% [32]. This indicates that despite high ITN use, An. arabiensis mosquitoes are still obtaining human blood meals. One explanation is mosquitoes feeding prior to 10 p.m., when residents go to bed, but only approximately $14 \%$ of $A n$. arabiensis in Macha forage during this time [32]. An alternate explanation, explored in this study, is that the ITNs in use are not completely effective at preventing mosquito bites. This could be caused either by pyrethroid resistance in the An. arabiensis population, or by a loss of insecticide or holes in the LLINs. Therefore, our aim was to both investigate the insecticide susceptibility status of the An. arabiensis population in Macha, as well as to evaluate the condition of LLINs distributed in Macha, in order to determine their potential efficacy after two years of typical use in the community.

\section{Methods}

\section{Study area}

This study was conducted at the Johns Hopkins Malaria Research Institute's field station in Macha, Southern 
Province, Zambia. It is located at $16.39292^{\circ} \mathrm{S}, 26.79061^{\circ}$ $\mathrm{E}$, at an elevation of approximately 1,100 meters above sea level. The habitat around the Malaria Institute at Macha (MIAM) field station is characterized as Miombo woodland. Macha inhabitants are primarily subsistence farmers living in village areas under a headman. ITNs were collected from the villages of Chidakwa and Lupata, each $3 \mathrm{~km}$ from the research facility and Macha Mission Hospital. Each household in these villages consists of a collection of small houses constructed of mud or brick, with thatch or steel roofs. Previous surveys conducted during the rainy season have determined that approximately $75 \%$ of inhabitants in these villages slept under an ITN the previous night [32]. Anopheles arabiensis females were collected for insecticide resistance assays in Chidakwa, located $3 \mathrm{~km}$ from Macha hospital, and in Namwala, located $80 \mathrm{~km}$ north of Macha. Mosquitoes were collected during January 2010.

\section{Anopheles arabiensis colony}

The MIAM An. arabiensis colony kept at Macha was characterized for insecticide susceptibility, and used as a baseline against which to compare field mosquitoes. This colony was established in February 2008 from mosquitoes collected in the Macha area. Because the colony has been through a strong bottleneck, and is kept free from any source of insecticide, it is presumed to be susceptible to most insecticides.

\section{F1 field mosquitoes}

Anopheline mosquitoes were collected by outdoor human landing catch [33] and kept in paper cups with sugar solution. Mosquitoes were identified morphologically [34], and those identified as An. gambiae s.l. were kept in insectary conditions $\left(28^{\circ} \mathrm{C}, 80 \%\right.$ relative humidity) for blood feeding and oviposition. Mosquitoes were offered a blood meal from a mouse, then kept in individual snap-cap vials with $10 \%$ sucrose pads and damp filter paper for oviposition. Ethical approval for mouse feeds was given by the Johns Hopkins University Animal Care and Use Committee (Protocol Number: GP10H223). After oviposition, parental mosquitoes were killed by freezing, and DNA was extracted to identify species [35]. After parental mosquitoes were positively identified as An. arabiensis, F1 egg batches were pooled. Larvae were reared to adulthood in the insectary, and 35 day old mixed-sex adults were used in bioassays.

\section{CDC bottle bioassay}

Adult mosquitoes were tested for insecticide susceptibility against permethrin, deltamethrin, DDT, and malathion (Sigma Aldrich, St. Louis, MO) using CDC bottle bioassays $[36,37]$. DDT was tested due to its use in indoor residual spraying programmes, permethrin and deltamethrin due to their use in LLINs and IRS, and malathion because it is used agriculturally. Briefly, the inside of 250-mL glass Wheaton bottles were coated with doses of insecticide diluted in acetone, including one control with acetone only. Dosages were measured as $\mu \mathrm{g} /$ bottle. The following dosages were tested against colony An. arabiensis: 30, 45, 60, and $75 \mu \mathrm{g} /$ bottle permethrin; 50, 100, 150, and $200 \mu \mathrm{g} /$ bottle DDT; 5, 10, 15, and $20 \mu \mathrm{g} /$ bottle deltamethrin; 25, 50, 100, and $150 \mu \mathrm{g} /$ bottle malathion. After the acetone had fully evaporated, 20-25 mosquitoes were introduced by aspiration. Knock-down was recorded at 15 minute intervals for three hours. After three hours, mosquitoes were removed from the bottles, separated into alive and knocked-down, and kept in separate paper cups with $10 \%$ sucrose solution for 24 hours in the insectary, after which they were scored as alive or dead. Diagnostic dosages established from these experiments were used to test the field population.

\section{Novel ITN susceptibility assay}

Netting from a deltamethrin-treated Permanet ${ }^{\circledR}$ LLIN was used to line the inside of a one-gallon cardboard container. A container lined with untreated netting material was used as a control for mortality. Twenty An. gambiae sensu strictu Keele laboratory colony mosquitoes were aspirated into each container and exposed to LLIN material for five minutes, after which they were gently shaken out into a cage and aspirated into labeled paper cups. They were then held for 24 hours in the insectary with $10 \%$ sucrose pads. After 24 hours, mosquitoes were categorized as dead or alive. Because pyrethroid insecticides cause mosquitoes to shed legs, which can negatively impact survival, live mosquitoes were further sorted into groups with 1-3 or 4-6 legs. This assay causes $100 \%$ mortality for deltamethrin-susceptible mosquitoes after 24 hours. The ITN assay was also compared against CDC bottle bioassays to determine mean time to knock down in both susceptible homozygous and $k d r$ L1014F and L1014S mosquitoes (Rebecca Trout-Fryxell and Anton Cornell, unpublished data), and has been used to characterize deltamethrin-resistant Culex quinquefasciatus mosquitoes in Macha [38].

Like the WHO cone assay [39], this assay exposes mosquitoes to insecticide-treated netting material. However, in the WHO assay, mosquitoes are able to land on the cone and thus avoid the ITN material. This test is more conservative because all surfaces are covered in netting, forcing mosquitoes to land on the netting and become exposed to insecticide.

\section{Knockdown resistance $(k d r)$ diagnostic}

DNA was extracted from both colony and field samples of $A n$. arabiensis using a modified salt-extraction [31] 
and was used to genotype samples for the $k d r$ allele, using the PCR-based method of Tripet et al [40].

\section{LLIN collection}

LLINs were collected in February 2009 from households that were known to have received Permanet ${ }^{\circledR} 2.0$ LLINs from our field team during the ITN distribution in February 2007. Owners of the nets were surveyed about the nets and their use. Questions included: 1) How old is this net? 2) How many times have you washed the net? 3) How many/what size are the windows in the house where this net was hung? 4) What kind of fuel is burned in the house? 5) Was the net retreated? Households were provided with new Permanet ${ }^{\mathbb{B}} 3.0$ nets to replace the nets taken. Nets were cataloged by their color and amount of wear, represented by the number of holes in five size categories $(>1 \mathrm{~cm}, 1-5 \mathrm{~cm}, 5-15 \mathrm{~cm}, 15-30 \mathrm{~cm}$, $>30 \mathrm{~cm}$ ). Nets were identified by the household number from which they were collected.

\section{Residual deltamethrin testing}

$20 \mathrm{~cm} \times 20 \mathrm{~cm}$ samples were cut from the top, middle, and bottom of each net for residual deltamethrin quantitation by gas chromatography. Samples were weighed and placed in a $20 \mathrm{~mL}$ scintillation vial. $20 \mathrm{~mL}$ xylene and $150 \mu \mathrm{l}$ internal standard solution $(5 \mathrm{~g} / \mathrm{mL}$ lambdacyhalothrin in xylene) was added to each scintillation vial, which was capped and gently shaken. Vials were placed in a $90^{\circ} \mathrm{C}$ water bath for 2 hours, then sonicated for 15 minutes. After cooling, $50 \mu \mathrm{l}$ of each sample solution was mixed with $950 \mu \mathrm{l}$ xylene and transferred to an auto-injection vial. Sample solutions were injected into the gas chromatography system, alternating with a calibration solution of standard-grade deltamethrin.

\section{Field LLIN mosquito survival assays}

Additional swatches were cut from the middle section of each bed net for mosquito survival bioassays. This assay was conducted similarly to the ITN susceptibility assay, using susceptible colonized $A n$. gambiae s.s. Keele strain mosquitoes. Three trials were performed with each LLIN sample, for a total of 60 mosquitoes tested on each net.

\section{Statistics}

The difference in deltamethrin concentration between top, middle, and bottom of bed net samples was tested by two-way ANOVA without replication. The effects of washing, wear on nets (measured by holes), type of fuel burned, and number of windows were analyzed as follows: washing data was categorized as no washes (0 reported washes), few washes (1-3 reported washes), and many washes ( $\geq 4$ washes); the number of holes in each net was enumerated by size $(<1 \mathrm{~cm}, 1-5 \mathrm{~cm}, 5-15 \mathrm{~cm}$,
15-30 cm, and > $30 \mathrm{~cm}$ ) and the number of holes weighted by size was calculated to give a score for wear; nets were categorized as being in houses where either kerosene or an alternate fuel was burned (diesel, solar, flashlight, candles); and the number of windows was counted in each house, then categorized as few (1-3) or many (5-10). The concentration of deltamethrin from the middle of each net was log-transformed to give a normal distribution, and a multi-factorial ANOVA was performed with the above factors. Prior to all ANOVA analyses, data was tested for normality by the skewness/ kurtosis test and for equal variance by Bartlett's test.

The relationship between deltamethrin concentration in middle swatches of the ITN and mosquito survival bioassays was modelled by logistic regression, after correcting for control mortality by Abbott's equation. The logistic regression was used to determine the $\mathrm{LD}_{90}$ of deltamethrin for this assay. All analyses were done in STATA 11 [41].

\section{Results}

\section{CDC bottle bioassays}

The diagnostic dosage for a CDC bottle bioassay is one that allows for discrimination between susceptible and resistant individuals. Dosages that are too low will show false resistance, while dosages that are too high will obscure actual resistance. Therefore, the diagnostic dosage is the lowest dose at which knock-down or mortality is saturated: increasing the dosage shows no increase in the slope of the mortality curve. Typically, this also correlates with $100 \%$ mortality within 1 hour. The following diagnostic dosages were established for the MIAM An. arabiensis colony: $60 \mu \mathrm{g} /$ bottle permethrin, $100 \mu \mathrm{g} /$ bottle DDT, $20 \mu \mathrm{g} /$ bottle deltamethrin, and $100 \mu \mathrm{g} /$ bottle malathion (Figure 1). These doses were close to the suggested CDC bottle bioassay doses for Anopheles species [42], supporting the hypothesis that this colony is susceptible to these insecticides. Different doses of deltamethrin, as low as $5 \mu \mathrm{g} /$ bottle, had no effect on the slope of the mortality curve. Type I and II pyrethroids differ in their neurophysiological symptoms [43], and binding kinetics [20]. Deltamethrin, a Type II pyrethroid, is known to have high poisoning efficacy [44], so it is possible that its faster knockdown time made the effects of different doses indistinguishable in this assay. Therefore, $20 \mu \mathrm{g} /$ bottle was used to test the field population, as this is a typical dose for Anopheles mosquitoes [42]. For all dosages tested, there was $100 \%$ mortality by 3 hours, and no recovery at the 24 hour time point.

The F1 field population was $100 \%$ susceptible to permethrin and deltamethrin at the diagnostic dosages used (Figure 2). In the DDT trial, mortality was $95 \%$ at 1 hour, and 100\% mortality did not occur until 105 


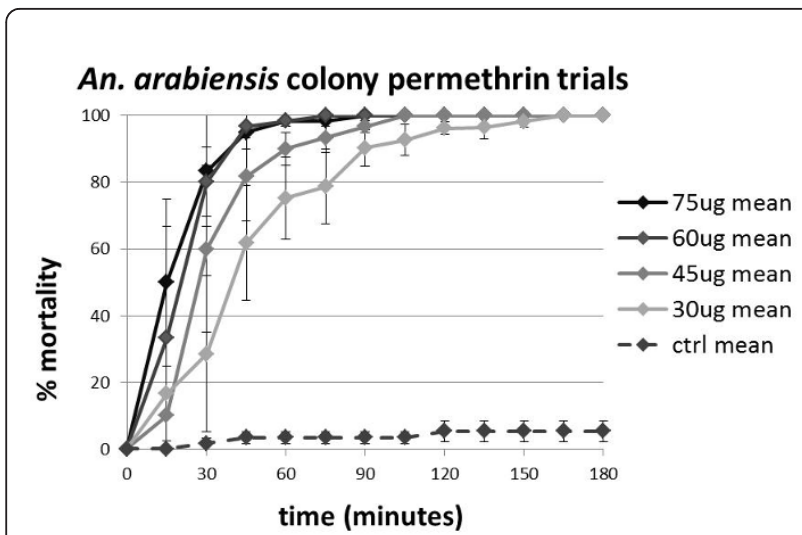

An. arabiensis colony deltamethrin trials

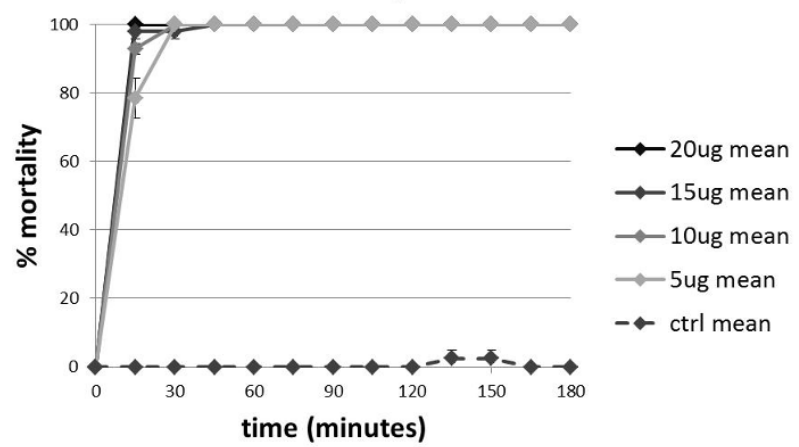

An. arabiensis colony DDT trials

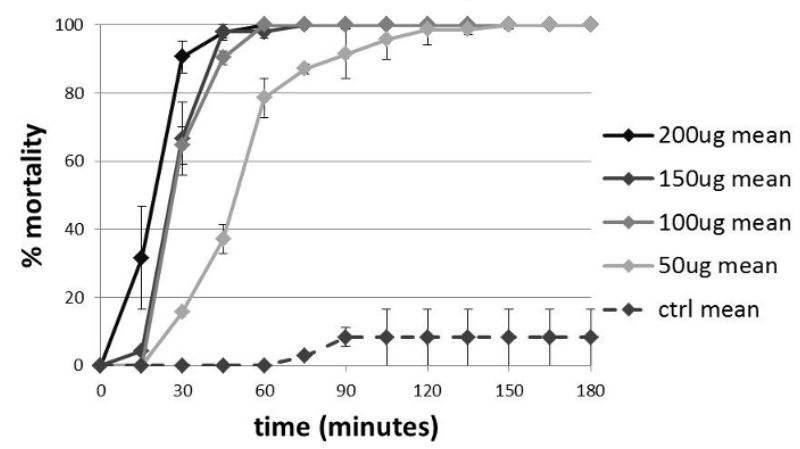

An. arabiensis colony malathion trials

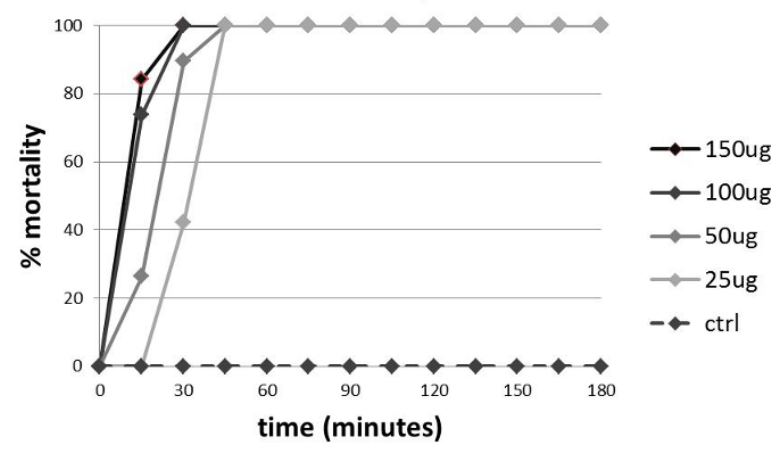

Figure 1 Mortality curves for CDC bottle bioassays performed on the MIAM An. arabiensis colony. In order to determine the diagnostic dosage, increasing concentrations of DDT, permethrin, deltamethrin, and malathion were used. The proper doses were determined to be: 100 $\mu \mathrm{g} / \mathrm{bottle} \mathrm{DDT}, 60 \mu \mathrm{g} / \mathrm{bottle}$ permethrin, $20 \mu \mathrm{g} / \mathrm{bottle}$ deltamethrin, and $100 \mu \mathrm{g} / \mathrm{bottle}$ malathion.

minutes (Figure 2). There was $100 \%$ mortality by 3 hours, and no recovery at the 24 hour time point. Due to a limited number of F1 mosquitoes and the priority of testing DDT, pyrethroids, and ITNs, F1 mosquitoes were not tested with malathion.

\section{ITN susceptibility assays}

At the 24 hour time point, colonized An. arabiensis showed $100 \%$ mortality when exposed to deltamethrintreated Permanet ${ }^{\circledR}$ material, versus $5 \%$ mortality with untreated material (Figure 3). In contrast, F1 field $A n$. arabiensis showed only $88 \%$ mortality, with $6 \%$ surviving with 1-3 legs and 6\% surviving with 4-6 legs. There was no mortality with untreated material, although $40 \%$ (2 out of 5) mosquitoes had 1-3 legs.

\section{$k d r$ genotyping}

In total, 50 colony An. arabiensis, 50 archived An. arabiensis from the field, and all 170 of the F1 An. arabiensis used in the bioassays were genotyped for $k d r$. All mosquitoes were homozygous wild-type, and the $k d r$ L1014F and L1014S alleles were not present in the population.

\section{LLIN sampling}

In total, 19 LLINs were collected from 16 households in Chidakwa and Lupata. All nets were currently hanging above beds in the houses when they were collected, indicating that they were actually in use. The median deltamethrin concentration varied depending on the location on the net from which the sample was taken, with $5.68 \mathrm{mg} / \mathrm{m}^{2}$ for swatches from the bottom of nets, $22.5 \mathrm{mg} / \mathrm{m}^{2}$ from the middle of nets, and $36.1 \mathrm{mg} / \mathrm{m}^{2}$ from the top of nets (Figure 4). The difference between the top, middle, and bottom of nets was significant $(\mathrm{p}<$ 0.0001, two-way ANOVA). Deltamethrin concentrations from swatches from the middle of the nets were therefore taken representative of the whole net, in order to simplify further statistical analyses.

\section{Factors relating to deltamethrin retention}

In the multi-factorial ANOVA, the only significant factor influencing deltamethrin concentration in bed nets was the number of reported washes $(p=0.001)$. Number of holes, which was a proxy for wear $(p=0.1608)$, kerosene burning in the hut $(\mathrm{p}=0.9311)$ and number of windows $(\mathrm{p}=0.9416)$ were not significant. There was 


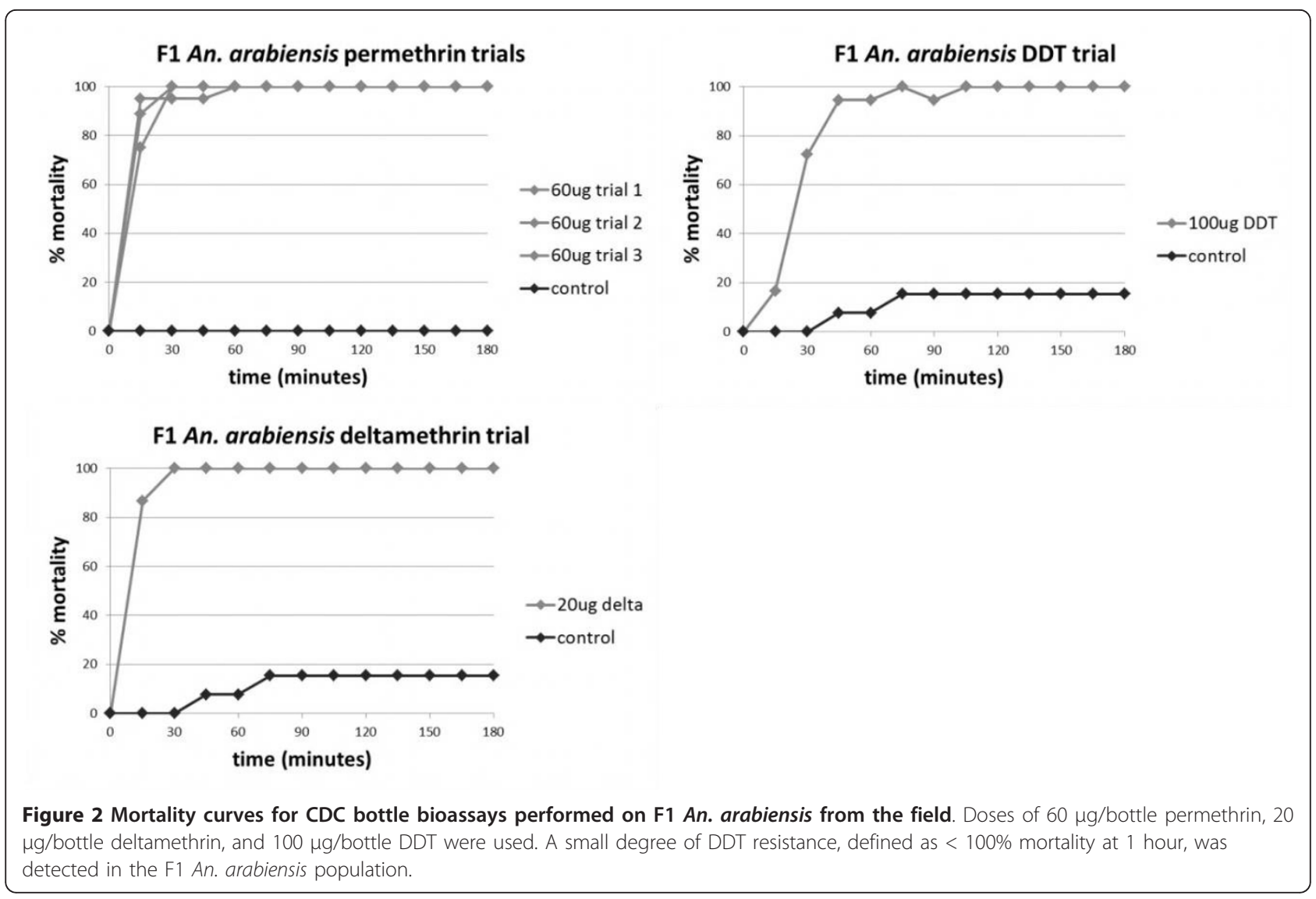

wide variation in the reported number of times the LLINs were washed, with a minimum of 0 times over the 2 year period to a maximum of 24 times, with a median of two washes. The median deltamethrin concentration varied between wash categories, with 39.4 $\mathrm{mg} / \mathrm{m}^{2}$ for those with no washes, $23.3 \mathrm{mg} / \mathrm{m}^{2}$ for those

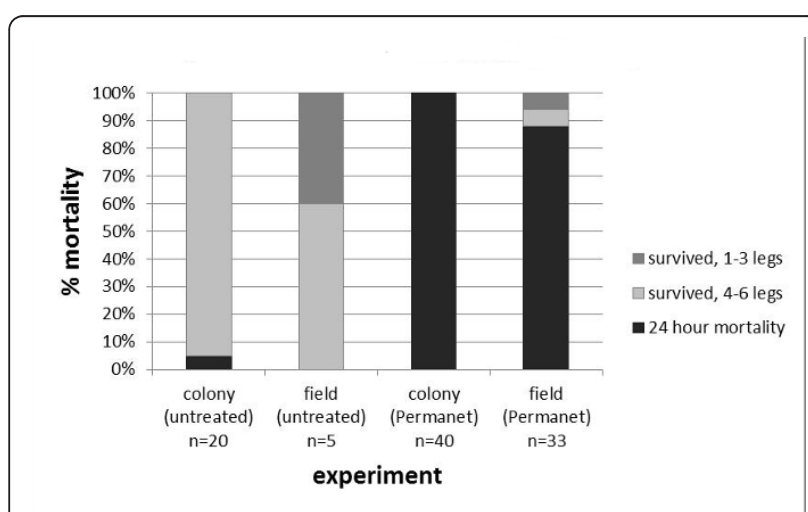

Figure 3 ITN bioassay survival. ITN survival assays were performed on MIAM An. arabiensis colony mosquitoes and F1 An. arabiensis from the field. Trials with untreated control netting and with deltamethrin-treated netting are shown. There was a small degree of resistance, defined as $<100 \%$ mortality at 24 hours, in the F1 field population. with 1 -3 washes, and $0.38 \mathrm{mg} / \mathrm{m}^{2}$ for those with 4 or more washes (Figure 5).

\section{Wear on LLINs}

There was wide variability in the amount of wear on the LLINs (Table 1). Of the 19 nets, five had no holes of

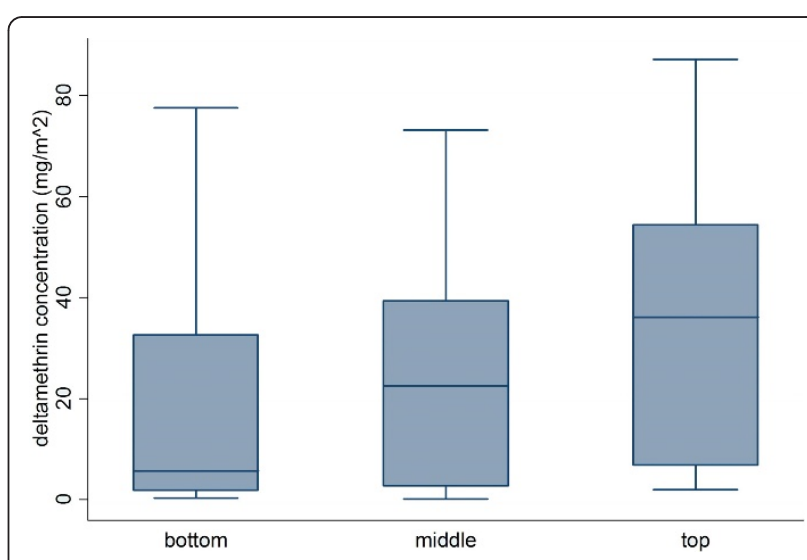

Figure 4 Distribution of deltamethrin in sections from the top, middle, and bottom of LLINs. Boxplots with median, range, quartiles, and outliers are shown. There were significantly lower residual deltamethrin concentrations in the middle and lower sections of the nets $(p<0.0001)$. 


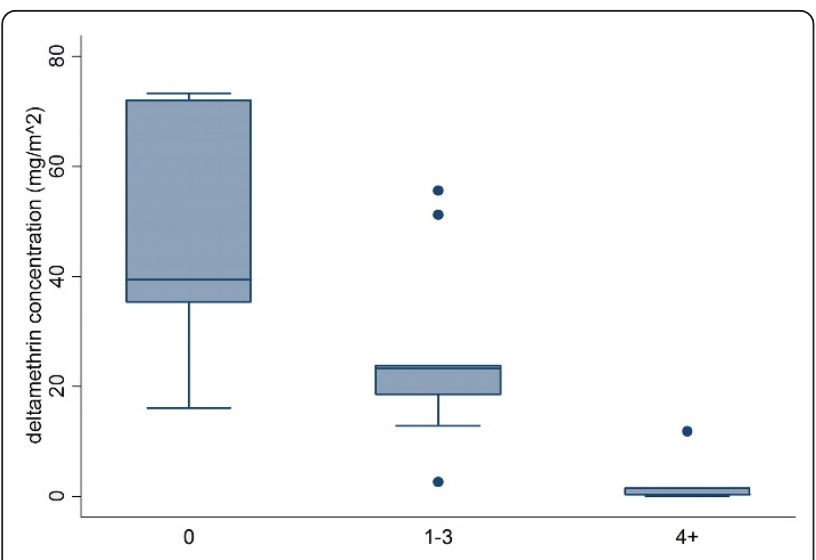

Figure 5 Deltamethrin concentration in the middle sections of nets, sorted by $\mathbf{0 , 1 - 3}$, and $4+$ washes. There was a significant correlation between number of washes and residual deltamethrin concentration $(p=0.001)$.

any size. The majority of holes were $1-5 \mathrm{~cm}$ in diameter, although five nets had holes greater than $15 \mathrm{~cm}$ in diameter, and one net had holes greater than $30 \mathrm{~cm}$ in diameter. Three of the nets had holes that had been mended by the owners.

Field LLIN mosquito survival bioassays

In the mosquito ITN survival bioassays, mortality ranged from $51 \%$ to $100 \%$, with $4.8 \%$ mortality for control mosquitoes (Figure 6). The majority of surviving mosquitoes had 1-3 legs, although six nets had mosquitoes that survived with 4-6 legs, and for one net, 9.5\% of all mosquitoes survived with 4-6 legs. For the negative control, $92 \%$ of all mosquitoes survived with 4-6 legs. The logistic model $f(x)=\frac{1}{1+e^{-\left(\beta_{0}+\beta_{1} x\right)}}$ was used to relate residual deltamethrin in the middle swatches of nets to mosquito survival in bioassays, with $\beta_{0}=-0.1253$ and $\beta_{1}=-0.0807$ (Figure 7). The model fit the observed data with $\mathrm{p}<$ 0.0001. Using this model, the $\mathrm{LD}_{90}$ for deltamethrin in this assay was calculated as $25.7 \mathrm{mg} / \mathrm{m}^{2}$.

\section{Discussion}

The results of the CDC bottle bioassays with the MIAM An. arabiensis colony indicate that it is susceptible to permethrin, DDT, deltamethrin, and malathion, at dosages near those used to test $A n$. gambiae s.s. It is also $100 \%$ susceptible to deltamethrin-treated material used in bed nets. Finally, the $k d r$ allele, which causes resistance to pyrethroids and DDT, is absent from the colony. Therefore, the colony is appropriate to use as a susceptible control which can be used as a baseline against which to compare field populations. It can also be used for other applications, such as testing LLINs from the field for efficacy. This is the first report on this novel An. arabiensis colony, and the first such colony originating from this region.

Table 1 Number of holes in each LLIN, by diameter (centimeters)

\begin{tabular}{|c|c|c|c|c|c|}
\hline Household ID & $\begin{array}{l}\text { very small holes } \\
(<1 \mathrm{~cm})\end{array}$ & $\begin{array}{l}\text { small holes } \\
(1-5 \mathrm{~cm})\end{array}$ & $\begin{array}{c}\text { medium holes } \\
(5-15 \mathrm{~cm})\end{array}$ & $\begin{array}{l}\text { large holes } \\
(15-30 \mathrm{~cm})\end{array}$ & $\begin{array}{l}\text { very large holes } \\
(>30 \mathrm{~cm})\end{array}$ \\
\hline $\mathrm{HH} 1-\mathrm{H} 2$ & & & 2 & 1 & \\
\hline HH3-H5 & & 2 & & 1 & \\
\hline HH3-H8 & & 3 & & & \\
\hline HH4-H5 & & 7 & 1 & & \\
\hline $\mathrm{HH} 5-\mathrm{H} 1$ & & & 1 & & \\
\hline $\mathrm{HH} 6-\mathrm{H} 2$ & & 4 & 3 & & \\
\hline \multicolumn{6}{|l|}{$\mathrm{HH} 12-\mathrm{H} 3$} \\
\hline \multicolumn{6}{|l|}{$\mathrm{HH} 13-\mathrm{H} 2$} \\
\hline \multicolumn{6}{|l|}{$\mathrm{HH} 16-\mathrm{H} 2$} \\
\hline $\mathrm{HH} 19-\mathrm{H} 7$ & & 10 & 2 & & \\
\hline HH55-H8 & & 2 & 5 & 2 & 2 \\
\hline HH58-H1 & 2 & & & 1 & \\
\hline $\mathrm{HH70-H4}$ & 15 & 10 & 1 & & \\
\hline \multicolumn{6}{|l|}{$\mathrm{HH} 75-\mathrm{H} 4$} \\
\hline \multicolumn{6}{|l|}{ HH75-H5 } \\
\hline HH79-H1 & & 9 & 2 & 4 & \\
\hline HH81-H1 & & 1 & & & \\
\hline $\mathrm{HH} 90-\mathrm{H} 1$ & & 1 & 1 & & \\
\hline $\mathrm{HH} 90-\mathrm{H} 2$ & & & 1 & & \\
\hline Total & 17 & 49 & 19 & 9 & 2 \\
\hline
\end{tabular}




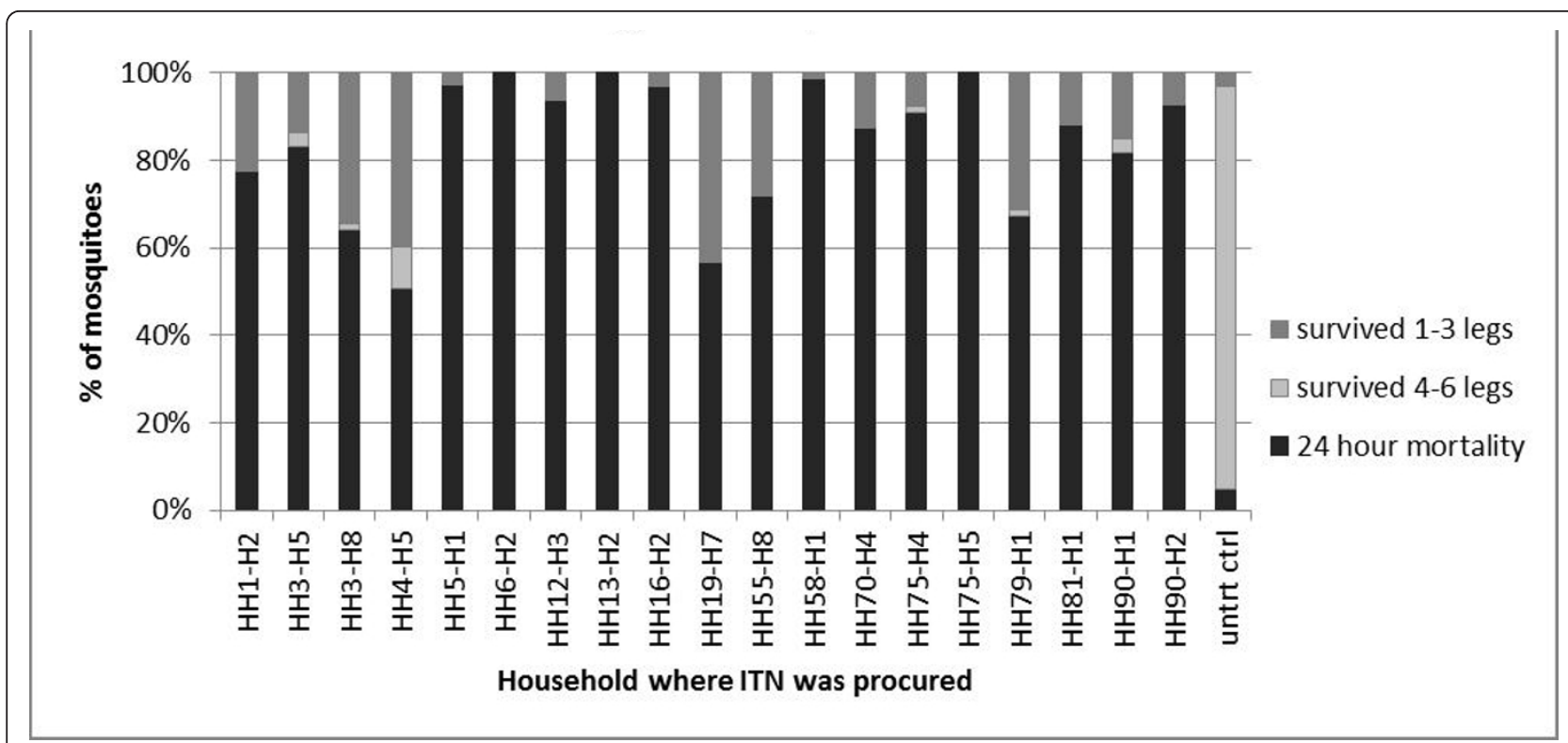

Figure 6 An. gambiae s.s. survival after exposure to netting obtained from LLINs in the field. Outcomes are mortality after 24 hours, survival with 1-3 legs, or survival with 4-6 legs.

The F1 field population of An. arabiensis appears to be completely susceptible to permethrin and deltamethrin, when tested in bottle bioassays. However, the mortality curve for the bottle bioassay with DDT shows slight resistance, and $12 \%$ of the mosquitoes tested survived after exposure to ITNs. It is possible that this test is more sensitive to lesser degrees of resistance in the mosquito. Because the $k d r$ allele, which confers targetsite resistance, is absent from the population, any resistance in this population is hypothesized to be mediated

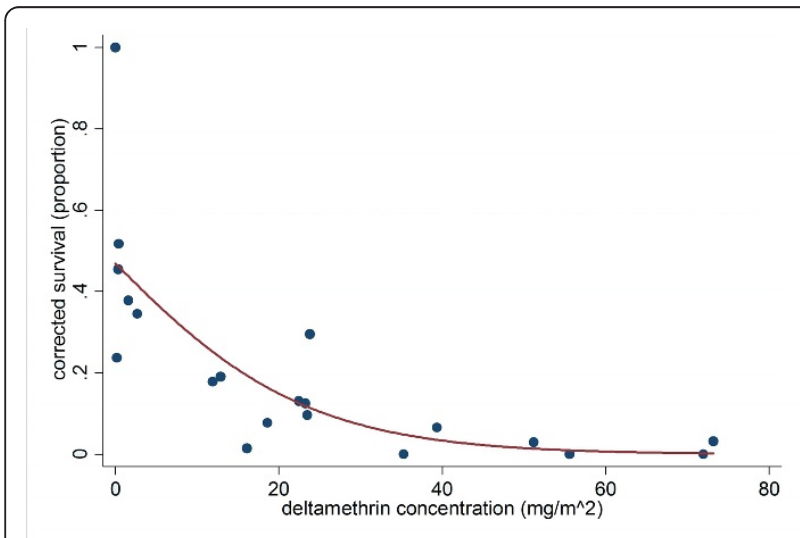

Figure 7 Logistic regression: deltamethrin vs. mosquito survival in bioassays. The relation between deltamethrin concentration in swatches of LLIN material and corrected mosquito survival in bioassays can be modelled as a logistic regression, using the function $f(x)=1 /\left(1+e^{0.1253+0.0807 x}\right)(p<0.0001)$. The $L D_{90}$ of deltamethrin-treated LLIN material is calculated as $25.7 \mathrm{mg} / \mathrm{m}^{2}$. by metabolic detoxification. Metabolic detoxification is typically caused by gene amplification and transcriptional upregulation $[18,45]$, making it more difficult to assay than $k d r$, which is a single nucleotide change. However, it can be assayed by detoxification enzyme activity assays [42]. Future studies will need to include characterization of the metabolic detoxification enzyme activity of this population of mosquitoes.

In contrast to previous research that focused on washing nets in a laboratory or tightly controlled field setting, this study examined how well LLINs performed in a real-world field setting, with no control or supervision over the behavior of the villagers using the bed nets. Therefore a wide range in variables were observed, such as the number of times a bed net was washed, how it was washed, UV exposure, handling and wear of the bed net, exposure to dust and soot, and other variables that may affect insecticide retention.

There was obvious patchiness in the distribution of residual deltamethrin in bed nets, with swatches from the bottom of the nets having the lowest concentration of insecticide. If ITNs are used improperly or not tucked under a mattress, mosquitoes and other biting insects can fly underneath the net, negating its effect as a physical barrier. Appropriate insecticide treatment, however, can either prevent this occurrence by spatial repellency, or cause the mosquito to die within 24 hours, preventing the spread of disease. If the bottom sections of nets do not retain enough residual insecticide, they may no longer have this protective effect. Similarly, holes and wear can cause nets to fail as a protective barrier, 
leaving only the effect of insecticide. If holes are very large, as was the case for some nets in this study, a mosquito can easily enter with very little searching, limiting the amount of insecticide contact, and again negating the ITN's protective effect.

ITNs, in addition to killing mosquitoes via insecticide, also function as a barrier to prevent bites. Holes in the net can undermine this function, particularly when residual deltamethrin concentrations in the net are too low to efficiently kill mosquitoes. It is therefore important to continue monitoring not only the concentration of insecticide in nets, but also their quality. A study in Kenya found that $40 \%$ of ITNs currently being used for malaria prevention were of poor quality due to the number of holes [46]. Although some of the nets in Macha had unacceptably large holes, a portion of them were completely intact after two years of use. The differences in wear between nets indicate that variation in how owners use nets can greatly affect bed net lifespan. It is possible that better education about how nets function to prevent malaria transmission by mosquitoes may help owners prioritize maintenance of their bed nets.

In this study, the greatest factor in loss of residual deltamethrin was the number of times the net was washed. It is possible that the other factors (wear, smoke, UV exposure) had an effect, but that it was not statistically significant due to the sample size, or that the proxies used to measure them did not accurately reflect the impact of those factors. Regardless, it is clear that the number of washes is not the only cause of deltamethrin loss. For example, some nets had never been washed, yet lost up to $30 \%$ of the residual insecticide from the top of the net, and up to $90 \%$ from the bottom of the net. It is possible that this variation was caused by the aforementioned factors, or by initial variation in the nets themselves. Nets that were washed at least once may have had additional variability in loss of deltamethrin, caused by differences in washing and drying methods between households.

The ITN survival bioassay used in this study is more conservative than the WHO cone test, because mosquitoes are forced to land on deltamethrin-treated netting, rather than having an untreated plastic surface available for resting. Despite this, some LLINs still had high survivorship when tested with the susceptible $A n$. gambiae s.s. colony. For six of the nets, there was less than $80 \%$ mortality, and for six nets a proportion of mosquitoes survived with four to six legs. However, as with other characteristics, there were some nets that still retained high efficacy even after two years of use-three of the nets had $100 \%$ mortality against $A n$. gambiae s.s. The logistic model showed that LLINs with deltamethrin concentrations as low as $25.7 \mathrm{mg} / \mathrm{m}^{2}$ maintained a $90 \%$ mortality rate in the ITN susceptibility assays, which is extremely close to the threshold given by the manufacturer [9].

This study demonstrates the importance of education as a major component of vector control campaigns. Although the initial variation in insecticide concentration in the nets is unknown, Some of these bed nets retained sufficient insecticide after two years to be as efficacious as new, unused ITNs. Clearly, it is possible for these nets to perform in the field nearly as well as they perform in the lab. Educating communities about proper care of their LLINs (how frequently to wash them, how to properly use the net as a physical as well as chemical barrier, the importance of mending holes and keeping the net away from sunlight), could help provide them with truly long-lasting treated nets.

It is unclear whether the slight pyrethroid and DDT resistance in the $A n$. arabiensis population will have an effect on vector control measures. If LLINs used in Macha are intact and have a full dose of residual deltamethrin, it is unlikely that this slight amount of resistance will have a great effect on LLIN efficacy. If, however, LLINs in Macha continue to deteriorate without replacement, insecticide resistance in the Macha $A n$. arabiensis population may play a larger role. Bed nets with lower residual deltamethrin concentrations may allow for feeding and select for higher insecticide resistance. Ongoing surveillance in Southern Zambia, as well as other areas of Zambia, will be necessary to control pyrethroid resistance, if it does emerge. Additionally, bed nets with more and larger holes require less probing by mosquitoes for them to obtain a blood meal while avoiding a toxic dose of insecticide. It is likely that low deltamethrin concentration in LLINs and holes in the nets are allowing $A n$. arabiensis mosquitoes to continue obtaining human blood meals in Macha despite the high use of ITNs.

\section{Conclusions}

In Macha, there are currently no explicit plans for a mass redistribution of LLINs in the near future. Although ITN roll-out campaigns have had an admirable impact and provided protection to a vast number of people, it is important for the organizations involved to consider how best to sustain these campaigns in the future. Ongoing assessments of ITN efficacy in different locations are necessary to determine when ITNs should be replaced, and plans should be in place for how best to prepare for ITN failure. There have been several cases of countries with successful malaria control programmes where a dramatic resurgence of malaria cases was seen when control measures ceased, such a Sri Lanka [47], Zimbabwe [48], and Peru [49]. In particular, waning immunity due to decreased malaria rates can contribute to a resurgence after control fails. Only three 
years after the introduction of ITNs in Macha, ITN use has been correlated to decreased seropositivity for $P$. falciparum (Tamaki Kobayashi and William Moss, unpublished data). It is critical, therefore, that vector control measures be maintained to ensure the continuing success of malaria control in Macha.

\section{Acknowledgements and Funding}

The authors wish to thank Mulenga Musapa at MIAM for contributing An. arabiensis colony mosquitoes and helping to rear F1 field samples, Chris Kizito at JHSPH for contributing An. gambiae s.s. colony mosquitoes, the Cornel lab at U.C. Davis for advice on implementing the ITN susceptibility assay, Dwight Mount at the Centers for Disease Control for residual deltamethrin quantitation, and Gregory Glass at JHSPH for help with statistical analysis. This work was funded by an NIH T32 Al 007417 Training Grant to Laura Norris and JHMRI funding and NIH ICEMR funding to Douglas Norris.

\section{Authors' contributions}

LN and DN conceived the study and designed the experiments. LN carried out mosquito and LLIN collections, mosquito bioassays, and PCRs, and analyzed the data. LN and DN drafted and wrote the manuscript. Both authors have read and approved the final manuscript.

\section{Competing interests}

The authors declare that they have no competing interests.

Received: 1 June 2011 Accepted: 31 August 2011

Published: 31 August 2011

\section{References}

1. WHO: WHO World Malaria Report 2010 World Health Organization, Geneva; 2010, 136

2. Lines JD, Myamba J, Curtis CF: Experimental hut trials of permethrinimpregnated mosquito nets and eave curtains against malaria vectors in Tanzania. Med Vet Entomol 1987, 1:37-51.

3. Miller JE, Lindsay SW, Armstrong JR: Experimental hut trials of bednets impregnated with synthetic pyrethroid or organophosphate insecticide for mosquito control in The Gambia. Med Vet Entomol 1991, 5:465-476.

4. Phillips-Howard PA, Nahlen BL, Kolczak MS, Hightower AW, ter Kuile FO, Alaii JA, Gimnig JE, Arudo J, Vulule JM, Odhacha A, Kachur SP, Schoute E, Rosen DH, Sexton JD, Oloo AJ, Hawley WA: Efficacy of permethrin-treated bed nets in the prevention of mortality in young children in an area of high perennial malaria transmission in western Kenya. Am J Trop Med Hyg 2003, 68:23-29.

5. Alonso PL, Lindsay SW, Armstrong JR, Conteh M, Hill AG, David PH, Fegan G, de Francisco A, Hall AJ, Shenton FC, Greenwood BM, Conteh M, Cham K, Hill AG, David PH, Fegan G, Hall AJ: The effect of insecticidetreated bed nets on mortality of Gambian children. Lancet 1991, 337:1499-1502.

6. Takken W: Do insecticide-treated bednets have an effect on malaria vectors? Trop Med Int Health 2002, 7:1022-1030.

7. WHO: Guidelines for laboratory and field testing of long lasting insecticidal mosquito nets World Health Organization, Geneva; 2005.

8. Ordonez Gonzalez J, Kroeger A, Avina Al, Pabon E: Wash resistance of insecticide-treated materials. Trans R Soc Trop Med Hyg 2002, 96:370-375.

9. Permanet 2.0 Specifications. [http://www.vestergaard-frandsen.com/ permanet/permanet-2/specifications].

10. RBM: Global strategic plan: Roll Back Malaria 2005-2015 Geneva, Switzerland: Roll Back Malaria Partnership, World Health Organization; 2005.

11. Chizema-Kawesha E, Miller JM, Steketee RW, Mukonka VM, Mukuka C, Mohamed AD, Miti SK, Campbell CC: Scaling up malaria control in Zambia: progress and impact 2005-2008. Am J Trop Med Hyg 2010, 83:480-488.

12. Mouzin E, Mukonka VM, Kamuliwo M, Hamainza B, Mwanza M, Wamalume P, Jennings T, Miller J: Country Report No. 2, Focus on Zambia. Roll Back Malaria Progress and Impact Series Geneva: World Health Organization; 2011
13. Kroeger A, Skovmand O, Phan QC, Boewono DT: Combined field and laboratory evaluation of a long-term impregnated bednet, PermaNet. Trans R Soc Trop Med Hyg 2004, 98:152-155.

14. Lindblade KA, Dotson E, Hawley WA, Bayoh N, Williamson J, Mount D, Olang G, Vulule J, Slutsker L, Gimnig J: Evaluation of long-lasting insecticidal nets after 2 years of household use. Trop Med Int Health 2005, 10:1141-1150.

15. Graham K, Kayedi MH, Maxwell C, Kaur H, Rehman H, Malima R, Curtis CF, Lines JD, Rowland MW: Multi-country field trials comparing washresistance of PermaNet and conventional insecticide-treated nets against anopheline and culicine mosquitoes. Med Vet Entomol 2005, 19:72-83.

16. Fettene M, Balkew M, Gimblet C: Utilization, retention and bio-efficacy studies of PermaNet in selected villages in Buie and Fentalie districts of Ethiopia. Malar J 2009, 8:114.

17. Kilian A, Byamukama W, Pigeon O, Atieli F, Duchon S, Phan C: Long-term field performance of a polyester-based long-lasting insecticidal mosquito net in rural Uganda. Malar J 2008, 7:49.

18. Hemingway J, Hawkes NJ, McCarroll L, Ranson H: The molecular basis of insecticide resistance in mosquitoes. Insect Biochem Mol Biol 2004, 34:653-665.

19. Najera JA, Zaim M: Malaria vector control: decision making criteria and procedures for judicious use of insecticides Geneva, Switzerland: World Health Organization Pesticide Evaluation Scheme (WHOPES); 2005.

20. Soderlund DM, Knipple DC: The molecular biology of knockdown resistance to pyrethroid insecticides. Insect Biochem Mol Biol 2003, 33:563-577.

21. Martinez-Torres D, Chandre F, Williamson MS, Darriet F, Berge JB, Devonshire AL, Guillet P, Pasteur N, Pauron D: Molecular characterization of pyrethroid knockdown resistance $(\mathrm{kdr})$ in the major malaria vector Anopheles gambiae s.s. Insect Mol Biol 1998, 7:179-184.

22. Chen H, Githeko AK, Githure Jl, Mutunga J, Zhou G, Yan G: Monooxygenase levels and knockdown resistance $(\mathrm{kdr})$ allele frequencies in Anopheles gambiae and Anopheles arabiensis in Kenya. J Med Entomol 2008, 45:242-250.

23. Hargreaves K, Hunt RH, Brooke BD, Mthembu J, Weeto MM, Awolola TS, Coetzee M: Anopheles arabiensis and An. quadriannulatus resistance to DDT in South Africa. Med Vet Entomol 2003, 17:417-422.

24. Dabire KR, Diabate A, Namountougou M, Toe KH, Ouari A, Kengne P, Bass C, Baldet T: Distribution of pyrethroid and DDT resistance and the L1014F kdr mutation in Anopheles gambiae s.l. from Burkina Faso (West Africa). Trans R Soc Trop Med Hyg 2009, 103:1113-1120.

25. Yewhalaw D, Bortel W, Denis L, Coosemans M, Duchateau L, Speybroeck N: First evidence of high knockdown resistance frequency in Anopheles arabiensis (Diptera: Culicidae) from Ethiopia. Am J Trop Med Hyg 2010, 83:122-125.

26. Himeidan YE, Chen H, Chandre F, Donnelly MJ, Yan G: Short report: permethrin and DDT resistance in the malaria vector Anopheles arabiensis from eastern Sudan. Am J Trop Med Hyg 2007, 77:1066-1068.

27. Casimiro S, Coleman M, Hemingway J, Sharp B: Insecticide resistance in Anopheles arabiensis and Anopheles gambiae from Mozambique. J Med Entomol 2006, 43:276-282.

28. Verhaeghen K, Van Bortel W, Roelants P, Backeljau T, Coosemans M: Detection of the East and West African kdr mutation in Anopheles gambiae and Anopheles arabiensis from Uganda using a new assay based on FRET/Melt Curve analysis. Malar J 2006, 5:16.

29. Munhenga G, Masendu HT, Brooke BD, Hunt RH, Koekemoer LK: Pyrethroid resistance in the major malaria vector Anopheles arabiensis from Gwave, a malaria-endemic area in Zimbabwe. Malar J 2008, 7:247.

30. Larkin GL, Thuma PE: Congenital malaria in a hyperendemic area. Am J Trop Med Hyg 1991, 45:587-592.

31. Kent RJ, Thuma PE, Mharakurwa S, Norris DE: Seasonality, blood feeding behavior, and transmission of Plasmodium falciparum by Anopheles arabiensis after an extended drought in southern Zambia. Am J Trop Med Hyg 2007, 76:267-274.

32. Fornadel CM, Norris LC, Glass GE, Norris DE: Analysis of Anopheles arabiensis blood feeding behavior in southern Zambia during the two years after introduction of insecticide-treated bed nets. Am J Trop Med Hyg 2010, 83:848-853.

33. Beier JC: Vector incrimination and entomological inoculation rates. Methods Mol Med 2002, 72:3-11. 
34. Gillies M, Coetzee M: A Supplement to the Anophelinae of Africa South of the Sahara. Johannesburg: South African Institute for Medical Research; 1987.

35. Scott JA, Brogdon WG, Collins FH: Identification of single specimens of the Anopheles gambiae complex by the polymerase chain reaction. Am J Trop Med Hyg 1993, 49:520-529.

36. Brogdon WG, MCAllister JC: Simplification of adult mosquito bioassays through use of time-mortality determinations in glass bottles. J Am Mosq Control Assoc 1998, 14:159-164.

37. Bottle Bioassays: Evaluating Mosquitoes for Insecticide Resistance. [http://www.cdc.gov/ncidod/wbt/resistance/assay/bottle/index.htm]

38. Norris LC, Norris DE: Insecticide resistance in Culex quinquefasciatus mosquitoes after the introduction of ITNs in Macha, Zambia. Journal of Vector Ecology .

39. WHO: Test procedures for insecticide resistance monitoring in malaria vectors, bio-efficacy and persistence of insecticide treated surfaces Geneva, Switzerland 1997.

40. Tripet F, Wright J, Lanzaro G: A new high-performance PCR diagnostic for the detection of pyrethroid knockdown resistance kdr in Anopheles gambiae. Am J Trop Med Hyg 2006, 74:658-662.

41. Statacorp: Stata Statistical Software: Release 11. College Station, TX: Statacorp, LP; 2009

42. Authentication by Bioassay. [http://www.mr4.org/CDCtraininghtml/tabid/ 1410/Default.aspx].

43. Gammon DW, Brown MA, Casida JE: Two classes of pyrethroid action in the cockroach. Pesticide Biochemistry and Physiology 1981, 15:181-191.

44. Vais H, Williamson MS, Devonshire AL, Usherwood PN: The molecular interactions of pyrethroid insecticides with insect and mammalian sodium channels. Pest Manag Sci 2001, 57:877-888.

45. Hemingway J, Hawkes N, Prapanthadara L, Jayawardenal KG, Ranson H: The role of gene splicing, gene amplification and regulation in mosquito insecticide resistance. Philos Trans R Soc Lond B Biol Sci 1998 353:1695-1699.

46. Githinji S, Herbst S, Kistemann T, Noor AM: Mosquito nets in a rural area of Western Kenya: ownership, use and quality. Malar J 2010, 9:250.

47. Gramaccia G, Beale P: The recent history of malaria eradication and control. In Malaria. Edited by: Wernsdorfer W, McGregor I. Edinburgh, United Kingdom: Churchill Livingstone; 1988:1335-1378

48. Zimbabwe Ministry of Health: Zimbabwe National Health Profile 1998. the Ministry of Health and Child Welfare. Harare, Zimbabwe; 2001.

49. Guarda J, Asayag R, Witzig R: Malaria re-emergence in the Peruvian Amazon region. Emerg Infect Dis 1999, 5:209-215.

doi:10.1186/1475-2875-10-254

Cite this article as: Norris and Norris: Efficacy of long-lasting insecticidal nets in use in Macha, Zambia, against the local Anopheles arabiensis population. Malaria Journal 2011 10:254

\section{Submit your next manuscript to BioMed Central and take full advantage of:}

- Convenient online submission

- Thorough peer review

- No space constraints or color figure charges

- Immediate publication on acceptance

- Inclusion in PubMed, CAS, Scopus and Google Scholar

- Research which is freely available for redistribution

Submit your manuscript at www.biomedcentral.com/submit
Biomed Central 